\title{
Testing or Fault-Finding for Reliability Growth: A Missile Destructive-Test Example
}

\author{
D.P. Gaver, P.A. Jacobs \\ Department of Operations Research, Naval Postgraduate School, Monterey, \\ California 93943
}

Received July 1996; revised May 1997; accepted 14 May 1997

\begin{abstract}
A new piece of equipment has been purchased in a lot of size $m$. Some of the items can be used in destructive testing before the item is put into use. Testing uncovers faults which can be removed from the remaining pieces of equipment in the lot. If $t<m$ pieces of equipment are tested, then those that remain, $m_{t}=m-t$, have reduced fault incidence and are more reliable than initially, but $m_{t}$ may be too small to be useful, or than is desirable. In this paper models are studied to address this question: given the lot size $m$, how to optimize by choice of $t$ the effectiveness of the pieces of equipment remaining after the test. The models used are simplistic and illustrative; they can be straightforwardly improved. (C) 1997 John Wiley \& Sons, Inc.* Naval Research Logistics 44: 623-637, 1997
\end{abstract}

Keywords: reliability growth; Bayesian sequential analysis; Poisson process; destructive testing; how much testing is enough; operational testing

\section{PROBLEM SETTING}

A new piece of equipment has been produced, and is to be tested before being put into use. An example is a military missile. Ultimate testing is done destructively by firing shots. The objective is to send equipment to the field with as few (design) faults as possible, so testing is focused on finding faults and removing them; it will be assumed here that once a fault is discovered it can be removed by change of design or componentry, and hence that a mode of failure has been permanently removed from all remaining missiles. The problem: If missiles are bought in lots of $m$, and $t<m$ are tested, then those that remain, $m_{t}=m-t$, have reduced fault incidence and are more reliable (the lot or design has experienced "reliability growth"), but $m_{t}$ may be too small to be useful, or than is desirable.

We address two problems.

(a) Given the lot size, $m$, how to optimize the effectiveness or lethality of the missiles remaining after $t(<m)$ are tested by choice of $t$.

Correspondence to: D.P. Gaver

(C) 1997 John Wiley \& Sons, Inc. *This article is a US Government work and, as such, is in the public domain in the United States of America.

CCC 0894-069X/97/070623-15 
(b) In the light of a testing program of length $t$, how does $t$ depend upon $m$; or how does lot size affect the final product's quality, where quality measures the probability of overall success in use? This means that both reliability and other suitability measures are combined with accuracy and target destination probability and other effectiveness measures to obtain an overall success probability when the missile is fired. The focus is entirely on maximizing operational capability, given the lot size $m$. Other calculations can be made to address questions of final, after-test missile adequacy to meet military needs particularly when compared to alternative, e.g., currently employed, options. The question of characterizing the uncertainty with which such a comparison is made is not thoroughly addressed here.

Related issues arise in reliability growth testing; cf. Ascher and Feingold [1], Balaban [2], Barlow and Scheuer [3], Barr [4], Bhattacharyya, Fries, and Johnson [5], Calabria, Guida, and Pulcini [6], Fries [9], Gross and Kamins [10], Jayachadran and Moore [13], Mazzuchi and Soyer [14], Olsen [15], Pollock [16], and Woods [17]. However, in traditional reliability growth testing, there is no constraint on the number of tests allowed.

\section{INITIAL MATHEMATICAL MODEL}

Suppose a missile design initially contains $D_{0}$ potential bugs or faults. If present, each of these independently inactivates a missile flight with probability $p$, or does not operate detrimentally with probability $1-p$. It is a considerable simplification to assume that $p$ is the same for all fault/bug types, and that $p$ does not depend on flight time or other conditions, but this simplification allows a quick initial evaluation. Note that if $m$ missiles are built as described, never tested but fired, then the number, $S_{0}$, of (later) successful flights is, given $D_{0}$, distributed binomially with probability of success $(1-p)^{D_{0}}$; consequently, its expectation is

$$
E\left[S_{0} \mid D_{0}\right]=\operatorname{sm}(1-p)^{D_{0}},
$$

where $s$ is the probability that a missile with no serious faults survives and operates properly. Various other meaningful measures can also be evaluated.

\subsection{Testing}

Suppose $t$ missiles are test-fired. If some fail it is presumed that (a) the particular faults causing failure are identifiable and (b) they are successfully removed from the remaining missiles, leaving $m-t$ as yet unfired and potentially useful in actual operations. Furthermore, these are now more reliable, but there is obviously a tradeoff involved in the choice of $t$. Thus after $t$ are tested (2.1) turns into

$$
E\left[S_{t} \mid D_{t}\right]=s(m-t)(1-p)^{D_{t}},
$$

where $D_{t}$ is the number of potential faults remaining after $t$ test firings. It is assumed that we are only removing single "root-cause faults" that can themselves bring about missile failure, whereas there actually could be a complicated interlocking sequence of fault failures, and a postmortem could possibly identify them, leading to their simultaneous removal. This 
optimistic situation is disregarded here. We also represent, in the parameter $s$, the influence of nonremovable faults: items that simply fail but cannot be design-rectified. Existence of such can slow down the reliability growth process by stimulating search for the unattainable. For the present this bit of realism is ignored, as is the possibility that identification of a removable fault leads to replacement by an item of higher $p$-value than that replaced! The present model is optimistic in that a new item is essentially compatible with $s$, not changing it by much.

\subsection{Property of a Test of Fixed Length $t$}

In order to choose the test period $t$, one can compute the expected value of those that survive later (active, combat) flights. This entails removal of the condition on $D_{t}$ in (2.2); one can then pick the $t$-value so as to maximize that expectation. This is one answer to "how much is enough testing" in the present context.

Suppose $D_{0}$ bugs/faults are originally present, and we ask how many are present after time $t$. The probability that any one is still present is $(1-p)^{t}$; by independence $D_{t}$ is binomial:

$$
P\left\{D_{t}=k \mid D_{0}\right\}=\left(\begin{array}{c}
D_{0} \\
k
\end{array}\right)\left((1-p)^{t}\right)^{k}\left(1-(1-p)^{t}\right)^{D_{0}-k}
$$

with generating function

$$
E\left[z^{D_{t}} \mid D_{0}\right]=\left(z(1-p)^{t}+\left(1-(1-p)^{t}\right)\right)^{D_{0}} .
$$

In turn, the condition on $D_{0}$ can be removed; if $g_{D_{0}}(z)$ is the generating function of $D_{0}$, then

$$
E\left[z^{D_{t}}\right] \equiv g_{D_{0}}\left(1-(1-p)^{t}(1-z)\right)
$$

In Subsection 2.3 we consider Poisson-seeded potential faults. In Subsections 2.5 and 2.6 we consider potential faults having a discrete uniform distribution and a discrete uniform distribution with a random range.

\subsection{Potential Faults Are Poisson-Seeded}

If $D_{0}$ is assumed Poisson with mean $\lambda$, then directly it is seen that $D_{t}$ is Poisson with mean $\lambda(1-p)^{t}$, which has generating function

$$
E\left[z^{D_{t}}\right]=e^{-\lambda(1-p)^{t}(1-z)}
$$

and (2.2), the expected number of successful missions after testing for time $t$ (where $0 \leq$ $t \leq m)$ :

$$
E\left[S_{t}\right]=s(m-t) e^{-\lambda p(1-p)^{t}}
$$


Thus if all parameters (except $s$ ) are known, or estimated, we can discover the value of $t$ $=t_{\mathrm{opt}}(\mathrm{m})$ that maximizes the expected number of missiles sent to the field that will function properly in use. Thus we have an initial approach to a particular problem of predetermining test duration so as to "optimize" a candidate measure of mission success.

Note that the distribution of $D_{0}$ can be regarded as a Bayes prior on an unknown parameter. Then the prior's parameter $\lambda$ can be obtained by combining expert judgment and data on previous tested and fielded comparable systems. This prior can be updated with each test episode using Bayesian procedures. This approach is explored in Section 3.

\subsection{A Max-Min Policy for Poisson-Seeded Faults}

Suppose nature is malevolent and for any number of tests conducted will choose $p$ so as to minimize the expected number of successes after performing $t$ tests. Let $s=1$, and assume $D_{0}$ is Poisson with mean $\lambda$. Let

$$
\begin{aligned}
f(p) & =\ln E\left[S_{t}\right] \\
& =\ln (m-t)-\lambda(1-p)^{t} p .
\end{aligned}
$$

Setting $(d / d p) f(p)=0$ and solving for $p$ results in the minimizing $p, p_{\min }=1 /(1+t)$. For this value of $p$

$$
E_{\min }\left[S_{t}\right]=(m-t) \exp \left\{-\lambda\left(\frac{t}{1+t}\right)^{t} \frac{1}{1+t}\right\}
$$

A criterion to choose the number of missiles to test is to pick the number of tests, $t$, that maximizes the above. We will call this policy the max-min policy. Such a number must be found numerically; it is of interest to compare its implications to those of other procedures.

\subsection{Alternate Potential Fault-Seeding Distribution}

It is plausible that if a system reaches later testing stages its propensity to contain many faults is low. Perhaps it is a modification of a previous design (an upgrade in military parlance) with only a few subsystems being candidates for serious faults. In this case the Poisson model, which admits arbitrarily many faults, might well be replaced by one that absolutely limits the number of active faults, so we investigate one of the simplest alternatives: a discrete uniform for $D_{0}$ over $(0,1,2, \ldots, \tilde{d})$. Other features remain as before.

The generating function of the discrete uniform is

$$
g_{D_{0}}(z)=\frac{1}{\tilde{d}+1} \sum_{d=0}^{\tilde{d}} z^{d}=\frac{1-z^{\tilde{d}+1}}{(\tilde{d}+1)(1-z)}
$$

so that

$$
E\left[S_{t}\right]=s(m-t) \frac{1}{\tilde{d}+1}\left[\frac{1-\left(1-(1-p)^{t} p\right)^{\tilde{d}+1}}{(1-p)^{t} p}\right]
$$


For numerical illustration we match means to that of the Poisson: $\tilde{d} / 2=\lambda$; this will not always be possible for small $\lambda$ since $\tilde{d} / 2 \geq \frac{1}{2}$. To compare the expected number of successful missions after testing using $t$ missiles for the Poisson fault-seeding model and the discrete uniform fault-seeding model, first consider the functions

$$
f_{E}(\tilde{d})=e^{-\tilde{d} / 2 a}=\left[1-\frac{\tilde{d}}{2} a+\frac{1}{2}\left(\frac{\tilde{d}}{2} a\right)^{2}-\cdots\right]
$$

and

$$
\begin{aligned}
f_{U}(\tilde{d}) & =\frac{1}{\tilde{d}+1}\left[\frac{1-(1-a)^{\tilde{d}+1}}{a}\right] \\
& =\frac{1}{\tilde{d}+1}\left[\frac{1}{a}\left[-\sum_{k=1}^{\tilde{d}+1}(-a)^{k}\left(\begin{array}{c}
\tilde{d}+1 \\
k
\end{array}\right)\right]\right] \\
& =\left[1-\frac{a \tilde{d}}{2}+a^{2} \frac{\tilde{d}(\tilde{d}-1)}{3 !}-\cdots\right],
\end{aligned}
$$

where $a=(1-p)^{t} p$. Thus, for a reasonably small $a=(1-p)^{t} p$, the expected number of successful missions after testing will be approximately the same for both models. Examine the numerical examples to follow to see that choice of the prior's specific form may be of secondary effect.

\subsection{Second Alternative for Fault-Seeding: Discrete Uniform with Random Range}

Suppose the previous setup is generalized by letting $\tilde{d}$, the range of the uniform, be another arbitrary discrete distribution, denoted $\left\{p_{k} ; k=0,1,2, \cdots\right\}$, e.g., but not necessarily Poisson. From (2.10)

$$
E\left[z^{D_{0}} \mid \tilde{d}\right]=\frac{1}{\tilde{d}+1} \frac{1}{1-z}\left(1-z^{\tilde{d}+1}\right)
$$

so

$$
\begin{aligned}
E\left[z^{D_{0}}\right] & =\frac{1}{1-z} \sum_{k=0}^{\infty} \frac{1-z^{k+1}}{k+1} p_{k} \\
& =\frac{1}{1-z} \sum_{k=0}^{\infty}\left[\int_{0}^{1} y^{k} p_{k} d y-\int_{0}^{z}(y)^{k} p_{k} d y\right] \\
& =\frac{1}{1-z}\left(\int_{z}^{1} p(w) d w\right),
\end{aligned}
$$

where $p(w)$ is the generating function of $\left\{p_{k}\right\}$. 
If $p(w)=e^{-\mu(1-w)}$, Poisson, then we get

$$
E\left[z^{D_{0}}\right]=\frac{1}{\mu(1-z)}\left(1-e^{-\mu(1-z)}\right)
$$

Now introduce $z=1-(1-p)^{t} p$ as before:

$$
E\left[(1-p)^{D_{t}}\right]=\frac{1}{\mu(1-p)^{t} p}\left(1-e^{-\mu p(1-p)^{t}}\right) .
$$

In order to match means, it is easiest to calculate

$$
E\left[D_{0} \mid \tilde{d}\right]=\tilde{d} / 2 \quad \text { so that } \quad \mu=2 E\left[D_{0}\right] .
$$

Thus, substituting (2.18) into (2.17) for $E\left[D_{0}\right]=\tilde{d} / 2$ and letting $a=p(1-p)^{t}$ results in

$$
E\left[(1-p)^{D_{t}}\right]=\frac{1}{\tilde{d} a}\left(1-e^{-\tilde{d} a}\right) .
$$

Comparing (2.19) and (2.12), it is seen that the expected number of successful missions after testing for the Poisson fault-testing model will be less than that for the discrete uniform with Poisson random range.

\subsection{Numerical Illustrations and Implications}

The meaning of (2.7) is revealed by studying some special cases. Figures 1 and 2 suggest that while the optimal value of test time certainly depends upon the parameter values, which are unknown or must be estimated, the optimum values remain in a relatively narrow range, at least over the range of parameter values studied. For what seems to be plausible values the numbers proposed for test are a smallish fraction of lot size, $m$. There is a helpful general insight: If $p$, the probability of fault activation, is relatively large, then a relatively small test tends to remove many potential faults, leaving the field reliability high, whereas a smaller $p$-value requires somewhat, but not substantially many, more, since leaving lowprobability offenders in place is relatively undamaging. The max-min policy for $\lambda=5$ and $m=100$ is to test 13 missiles with resulting expected number of successes 75.9. The maxmin policy for $\lambda=5$ and $m=500$ is to test 29 missiles with resulting expected number of successes 442.5. Figures 1 and 2 show that the max-min policy is (not surprisingly) somewhat conservative.

\section{SEQUENTIAL DESTRUCTIVE TESTING: MYOPIC BAYESIAN UPDATING}

With the exception of the max-min analysis given in Section 2.4, the previous analysis assumes that the design defect failure probability $p$ is known, or at least that its value may be satisfactorily approximated off-line from data for analogous systems, and then treated as "known."' Suppose, however, that data are available sequentially on the number of 


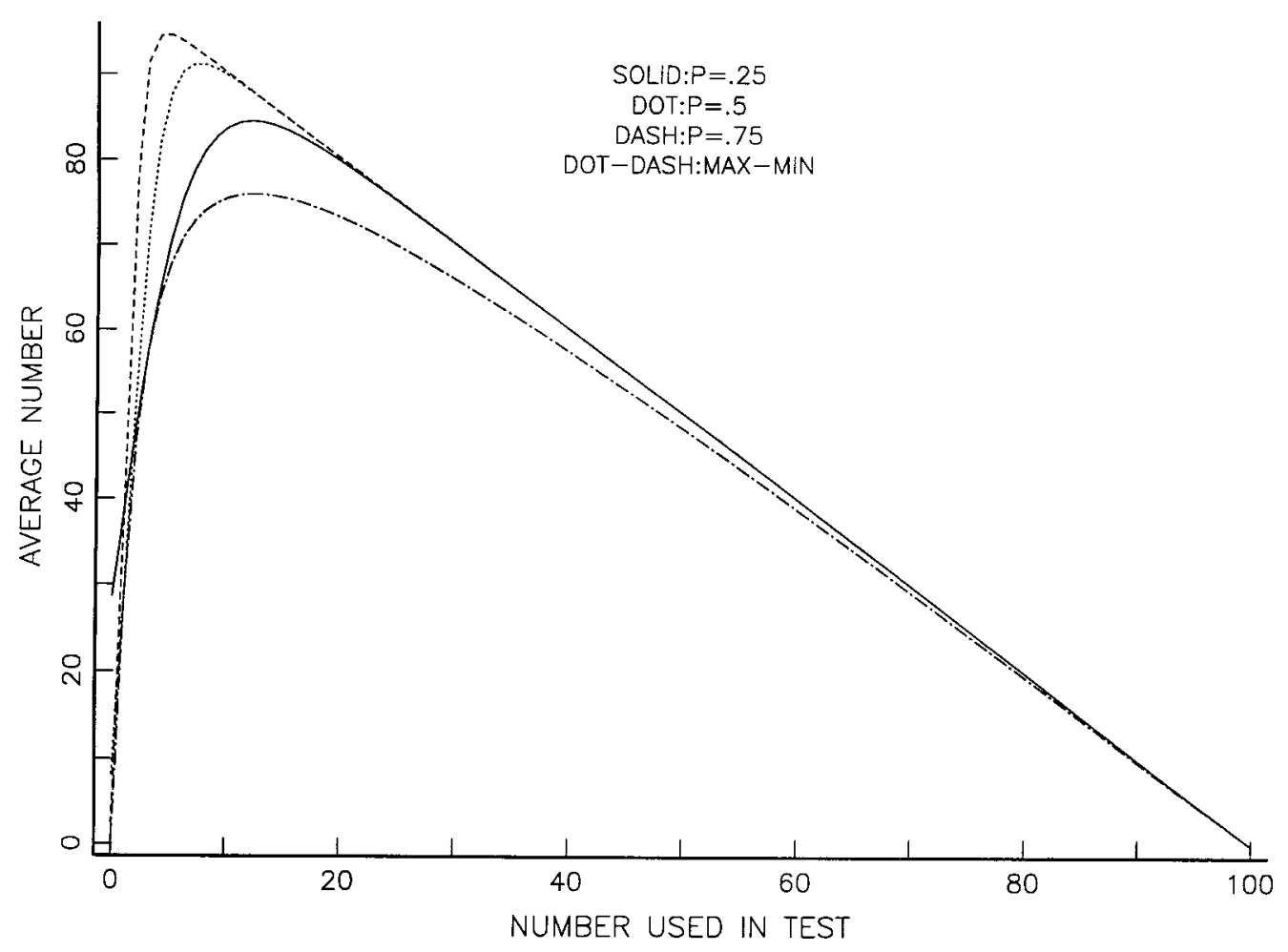

Figure 1. Average number of successes in remaining missiles. Poisson number of faults with mean LAM: total number of missiles $=100, \mathrm{LAM}=5$.

design defects that were revealed on an initial set of $t \in(1,2, \cdots)$ test firings of the missile in question. We show that such data can be used to provide a sequentially updated inference concerning $p$, and thus to decide when further testing is not justified. In Subsection 3.2 we discuss a criterion which compares the expected number of successes with the current posterior distribution of $p$ with that if we look forward to doing one more test. In Subsection 3.3 we discuss the criterion which is to test until all remaining (untested) missiles will be successful with a preselected probability. The problem we discuss is related, but not identical to, much work on sequential sampling and decision making. See in particular Chernoff and Ray [8] and Chernoff [7]; Yang, Wackerly, and Rosalsky [18] is also relevant.

The method described depends on these factors inherent in the basic model:

$D_{0}=$ the initial number of design defects that exist in the missile system,

$\theta=$ the probability a fault causes a failure in a missile,

$B_{1}=$ the number of faults discovered by the first test (assume all the faults are repaired upon discovery).

As previously, let $m$ be the total number of missiles. Assume 


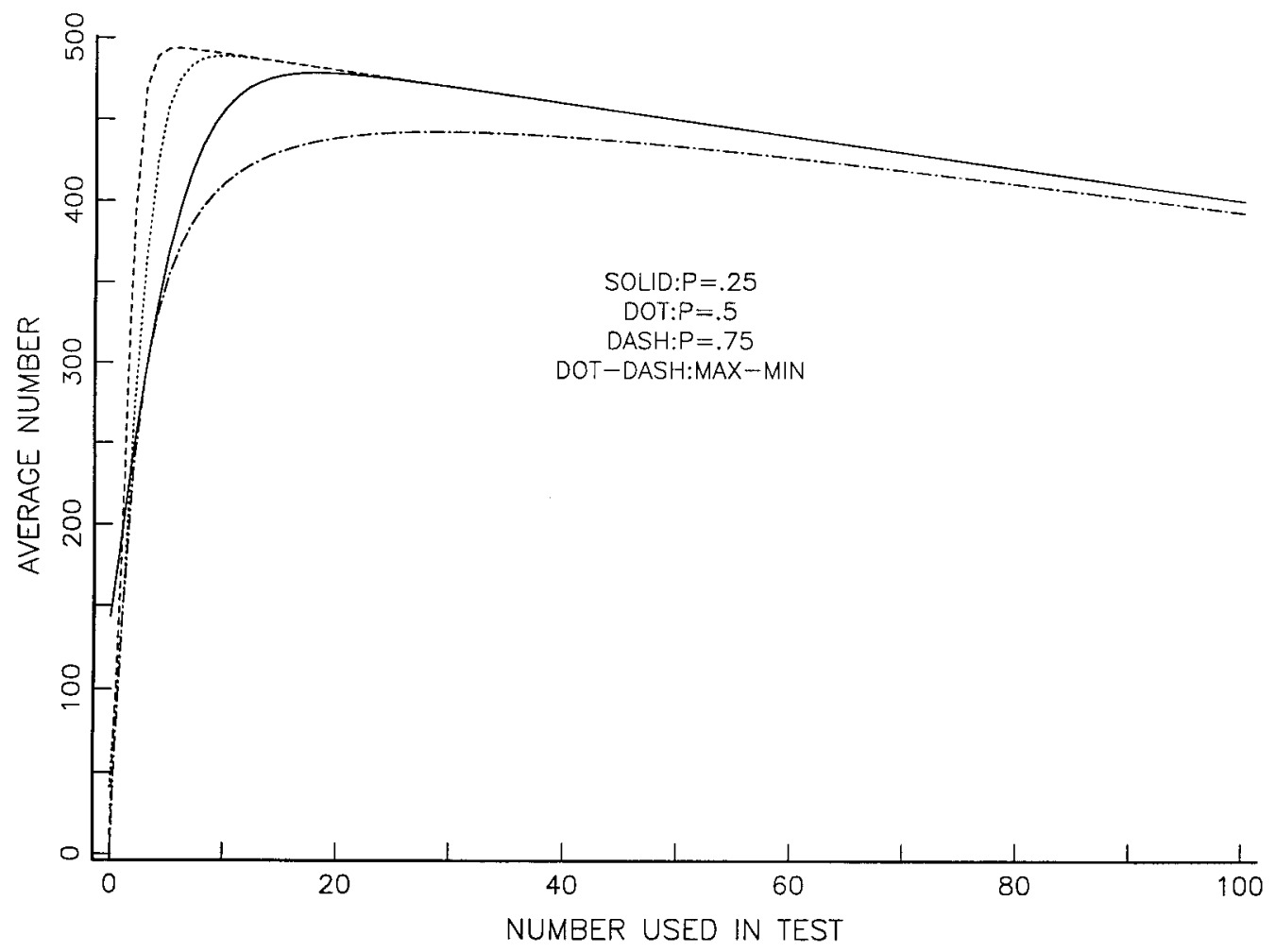

Figure 2. Average number of successes in remaining missiles. Poisson number of faults with mean LAM: total number of missiles $=500, \mathrm{LAM}=5$.

$$
\begin{gathered}
P\left\{B_{1}=b_{1} \mid D_{0}=d_{0}, \theta=p\right\}=\left(\begin{array}{c}
d_{0} \\
b_{1}
\end{array}\right) p^{b_{1}}(1-p)^{d_{0}-b_{1}}, \quad b_{1}=0, \ldots, d_{0}, \\
P\left\{D_{0}=d_{0}\right\}=\frac{e^{-\lambda} \lambda^{d_{0}}}{d_{0} !}, \quad d_{0}=0,1, \ldots, \\
P\{\theta \in d p\}=f(p) d p .
\end{gathered}
$$

Then

$$
\begin{aligned}
P & \left\{\theta \in d p, B_{1}=b_{1}, D_{0}=d_{0}\right\} \\
& =f(p) \frac{e^{-\lambda p}(\lambda p)^{b_{1}}}{b_{1} !} \frac{[\lambda(1-p)]^{d_{0}-b_{1}} e^{-\lambda(1-p)}}{\left(d_{0}-b_{1}\right) !} d p .
\end{aligned}
$$

Let $\bar{D}_{1}=D_{0}-B_{1}$, the number of remaining faults; then from (3.4) it follows that 


$$
P\left\{\theta \in d p, B_{1}=b_{1}, \bar{D}_{1}=\bar{d}_{1}\right\}=f(p) e^{-\lambda p} \frac{(\lambda p)^{b_{1}}}{b_{1} !} e^{-\lambda(1-p)} \frac{[\lambda(1-p)]^{d_{1}}}{\bar{d}_{1} !} d p
$$

and

$$
\begin{aligned}
P & \left\{\theta \in d p, \bar{D}_{1}=\bar{d}_{1} \mid B_{1}=b_{1}\right\} \\
& =K\left(b_{1}\right) f(p) e^{-\lambda p}(\lambda p)^{b_{1}} \frac{[\lambda(1-p)]^{\bar{d}_{1}} e^{-\lambda(1-p)}}{\bar{d}_{1} !} d p,
\end{aligned}
$$

where $K\left(b_{1}\right)=\left[\int_{0}^{1} f(p) e^{-\lambda p} p^{b_{1}} d p\right]^{-1}$. Similarly,

$$
\begin{aligned}
P\{\theta & \left.\in d p, B_{1}=b_{1}, B_{2}=b_{2}, \ldots, B_{k}=b_{k}, \bar{D}_{k}=\bar{d}_{k}\right\} \\
= & f(p) e^{-\lambda p} \frac{(\lambda p)^{b_{1}}}{b_{1} !} e^{-\lambda(1-p) p} \frac{[\lambda(1-p) p]^{b_{2}}}{b_{2} !} \times \cdots \\
& \times e^{-\lambda(1-p)^{k-1} p} \frac{\left[\lambda(1-p)^{k-1} p\right]^{b_{k}}}{b_{k} !} e^{-\lambda(1-p)^{k}} \frac{\left[\lambda(1-p)^{k}\right]^{\bar{d}_{k}}}{\bar{d}_{k} !} d p,
\end{aligned}
$$

where $\bar{D}_{k}=D_{0}-\left(B_{1}+\cdots+B_{k}\right)$, the number of remaining faults after $k$ tests.

\subsection{The Expected Number of Successes after $t$ Tests}

A missile is called a success if no faults occur during its launch or flight. Let $S_{0}=$ the number of successful missiles if no testing is done (no faults are fixed). Then

$$
\begin{aligned}
E\left[S_{0}\right] & =E\left[E\left[S_{0} \mid D_{0}, \theta\right]\right] \\
& =m \int_{0}^{1} \sum_{d_{0}=0}^{\infty}(1-p)^{d_{0}} e^{-\lambda} \frac{\lambda^{d_{0}}}{d_{0} !} f(p) d p \\
& =m \int_{0}^{1} \sum_{d_{0}=0}^{\infty} e^{-\lambda} \frac{[\lambda(1-p)]^{d_{0}}}{d_{0} !} f(p) d p \\
& =m \int_{0}^{1} e^{-\lambda p} f(p) d p .
\end{aligned}
$$

Suppose one test is done and $B_{1}=b_{1}$ faults are discovered and repaired; let $S_{1}$ be the number of successes in the remaining $(m-1)$ missiles:

$$
\begin{aligned}
E & {\left[S_{1} \mid B_{1}=b_{1}\right] } \\
& =(m-1) \int_{0}^{1} \sum_{d_{0}=0}^{\infty}(1-p)^{d_{0}} e^{-\lambda(1-p)} \frac{[\lambda(1-p)]^{d_{0}}}{d_{0} !} K\left(b_{1}\right) f(p) e^{-\lambda p}(\lambda p)^{b_{1}} d p \\
& =(m-1) \int_{0}^{1} e^{-\lambda(1-p) p} K\left(b_{1}\right) f(p) e^{-\lambda p}(\lambda p)^{b_{1}} d p .
\end{aligned}
$$


Similarly, if $k$ tests are conducted and $B_{i}$ faults are discovered and repaired on the $i$ th test, the expected number of successes in the remaining $(m-k)$ missiles is

$$
\begin{aligned}
& E\left[S_{k} \mid B_{1}=b_{1}, \ldots, B_{k}=b_{k}\right] \\
& \quad=(m-k) \int_{0}^{1} e^{-\lambda(1-p)^{k_{p}}} K\left(b_{1}, \ldots, b_{k}\right) f\left(p ; b_{1}, \cdots b_{k}\right) d p,
\end{aligned}
$$

where

$$
\begin{aligned}
f\left(p ; b_{1}, \cdots b_{k}\right)= & f(p) e^{-\lambda p} \frac{(\lambda p)^{b_{1}}}{b_{1} !} e^{-\lambda(1-p) p} \frac{[\lambda(1-p) p]^{b_{2}}}{b_{2} !} \times \cdots \\
& \times e^{-\lambda(1-p)^{k-1} p} \frac{\left[\lambda(1-p)^{k-1} p\right]^{b_{k}}}{b_{k} !}
\end{aligned}
$$

and

$$
K\left(b_{1}, \ldots, b_{k}\right)=\left[\int_{0}^{1} f\left(p ; b_{1}, \ldots, b_{k}\right) d p\right]^{-1}
$$

\subsection{The Expected Number of Successes after Looking Forward to Doing One More Test}

Before any tests are conducted consider the expected number of successes if one test were conducted. Let $S_{1}^{+}$be the number of successes using the remaining $(m-1)$ missiles. From (3.4)

$$
\begin{aligned}
E & {\left[S_{1}^{+} ; B_{1}=b, \theta \in d p\right] } \\
& =(m-1) f(p) \frac{e^{-\lambda p}(\lambda p)^{b}}{b !} \sum_{s=0}^{\infty}(1-p)^{s} \frac{[\lambda(1-p)]^{s}}{s !} e^{-\lambda(1-p)} d p \\
& =(m-1) f(p) \frac{e^{-\lambda p}(\lambda p)^{b}}{b !} \exp \{-\lambda(1-p) p\} d p .
\end{aligned}
$$

Thus,

$$
\begin{gathered}
E\left[S_{1}^{+} ; \theta \in d p\right]=(m-1) f(p) \exp \{-\lambda(1-p) p\} d p \\
E\left[S_{1}^{+}\right]=(m-1) \int_{0}^{1} f(p) \exp \{-\lambda(1-p) p\} d p
\end{gathered}
$$

Suppose $k$ tests have been done which resulted in $B_{1}=b_{1}, \ldots, B_{k}=b_{k}$ faults being discovered and repaired. Consider the expected number of successes if one more test were conducted. Let $S_{k}^{+}$be the number of remaining successes if another test is conducted. From (3.6) it follows that 


$$
\begin{aligned}
E & {\left[S_{k}^{+} ; B_{1}=b_{1}, B_{2}=b_{2}, \ldots, B_{k}=b_{k}, \theta \in d p\right] } \\
& =(m-(k+1)) f\left(p ; b_{1}, \ldots, b_{k}\right) d p \exp \left\{-\lambda(1-p)^{k} p\right\}
\end{aligned}
$$

where

$$
\begin{gathered}
f\left(p ; b_{1}, \ldots, b_{k}\right)=f(p) \frac{e^{-\lambda p}(\lambda p)^{b_{1}}}{b_{1} !} e^{-\lambda(1-p) p} \frac{[\lambda(1-p) p]^{b_{2}}}{b_{2} !} \times \cdots \\
\times e^{-\lambda(1-p)^{k-1} p} \frac{\left[\lambda(1-p)^{k-1} p\right]^{b_{k}}}{b_{k} !} \\
E\left[S_{k}^{+} \mid B_{1}=b_{1}, B_{2}=b_{2}, \ldots, B_{k}=b_{k}\right] \\
=\frac{(m-(k+1)) \int_{0}^{1} f\left(p ; b_{1}, \ldots, b_{k}\right) \exp \left\{-\lambda(1-p)^{k} p\right\} d p}{\int_{0}^{1} f\left(p ; b_{1}, \ldots, b_{k}\right) d p}
\end{gathered}
$$

A stopping rule might be to stop testing at $t_{B}$ tests where

$$
t_{B}=\min \left\{k: E\left[S_{k} \mid B_{1}=b_{1}, \ldots, B_{k}=b_{k}\right]>E\left[S_{k}^{+} \mid B_{1}=b_{1}, \ldots, B_{k}=b_{k}\right]+C\right\},
$$

where $C$ is a constant chosen by the analyst; possibly $C=0$. We will call this rule the (myopic) Bayes rule.

\subsection{The Probability of No Failure in the Remaining Missile Firings after Conducting $t$ Tests}

An alternative procedure is to test until all remaining (untested) missiles will be successful with a preselected probability. After $t$ tests, $0 \leq t \leq m$, the probability all the remaining missiles are successes is

$$
\begin{aligned}
P\left\{S_{m-t}=m-t\right\} & =\sum_{k=0}^{\infty} e^{-\lambda(1-p)^{t}} \frac{\left[\lambda(1-p)^{t}\right]^{k}}{k !}\left[(1-p)^{k}\right]^{m-t} \\
& =\exp \left\{-\lambda(1-p)^{t}\left[1-(1-p)^{m-t}\right]\right\}
\end{aligned}
$$

if $p$ and $\lambda$ are known.

If $\lambda$ is known but $p$ is not known, then

$$
\begin{aligned}
P & \left\{S_{m-t}=m-t, B_{1}=b_{1}, \ldots, B_{t}=b_{t}, D_{t}=k, \theta \in d p\right\} \\
& =f\left(p ; b_{1}, b_{2}, \ldots, b_{t}\right)(1-p)^{k(m-t)} e^{-\lambda(1-p)^{t}} \frac{\left[\lambda(1-p)^{t}\right]^{k}}{k !} d p
\end{aligned}
$$

where $f\left(p ; b_{1}, \ldots, b_{t}\right)$ is given by (3.14); with $k=t$

$$
\begin{aligned}
& f\left(p ; b_{1}, b_{2}, \ldots, b_{t}\right) \\
& \quad=f(p) e^{-\lambda p} \frac{(\lambda p)^{b_{1}}}{b_{1} !} e^{-\lambda(1-p) p} \frac{[\lambda(1-p) p]^{b_{2}}}{b_{2} !} \times \cdots \times e^{-\lambda(1-p)_{p}^{t-1}} \frac{\left[\lambda(1-p)^{t-1} p\right]^{b_{t}}}{b_{t} !} .
\end{aligned}
$$


Thus,

$$
\begin{aligned}
P & \left\{S_{m-t}=m-t, B_{1}=b_{1}, \ldots, B_{t}=b_{t}, \theta \in d p\right\} \\
& =f\left(p ; b_{1}, b_{2} \cdots, b_{t}\right) \exp \left\{-\lambda(1-p)^{t}\left[1-(1-p)^{m-t}\right]\right\} d p
\end{aligned}
$$

and

$$
\begin{aligned}
P & \left\{S_{m-t}=m-t \mid B_{1}=b_{1}, \ldots, B_{t}=b_{t}\right\} \\
& =K \int_{0}^{1} f\left(p ; b_{1}, \ldots, b_{t}\right) \exp \left\{-\lambda(1-p)^{t}\left[1-(1-p)^{m-t}\right]\right\} d p,
\end{aligned}
$$

where $K=\left[\int_{0}^{1} f\left(p ; b_{1}, \ldots, b_{t}\right)\right]^{-1}$.

A rule to stop testing may be to do $t_{P}$ tests, where

$$
t_{P}=\min \left\{k: P\left\{S_{m-k}=m-k \mid B_{1}=b_{1}, \ldots, B_{k}=b_{k}\right\}>\alpha\right\},
$$

where $\alpha=0.8$, or 0.9 , etc.

Numerical integration is required to carry out the above procedures, e.g., to evaluate integrals in (3.8), (3.9), (3.12), (3.15), and elsewhere. We have used Simpson's rule with up to 10th-order difference correction for a step size $h: 0.0001$ (cf. Hamming [11]) as implemented in A Graphical Statistical System, AGSS [12].

\subsection{Numerical Examples}

Figure 3 presents the expected number of successful missile flights after having conducted $t$ tests as a function of $t$ for a series of design fault discovery. There are three faults. One fault is discovered at test 3; one at test 4 ; and one at test 6 ; if no tests are conducted, the number of faults discovered is 0 . The prior distribution of the number of faults at time 0 is assumed to be a Poisson distribution with mean $\lambda=3$. The prior distribution for the probability of fault discovery, $\theta$, is uniform over $[0,1]$. The number of missiles in the lot $m=25$. The solid line plots the expected number of successes with no additional tests, (3.7) - (3.9). The dotted line plots the expected number of successes if one additional test is considered, (3.15). The dashed line plots the expected number of successes if a fixed number of tests are conducted for $\lambda=3$, and probability of discovery having the prior distribution, that is, from (2.7),

$$
E\left[S_{t}\right]=(m-t) \int_{0}^{1} e^{-\lambda p(1-p)^{t}} f(p) d p .
$$

A criterion which maximizes the expected number of successes for a fixed number of tests would stop testing after four tests. A criterion which stops testing when doing one more test would not result in a larger expected number of successes would also stop after test 4 . Both criteria would miss the one fault that does not appear until test 6 . The maxmin policy obtained using (2.9) for $m=25$ and $\lambda=3$ would also test four missiles.

Figure 4 displays plots of the probability that all $(m-t)$ remaining missiles are successes after conducting $t$ tests. There are 25 missiles initially. The prior distribution of the initial 


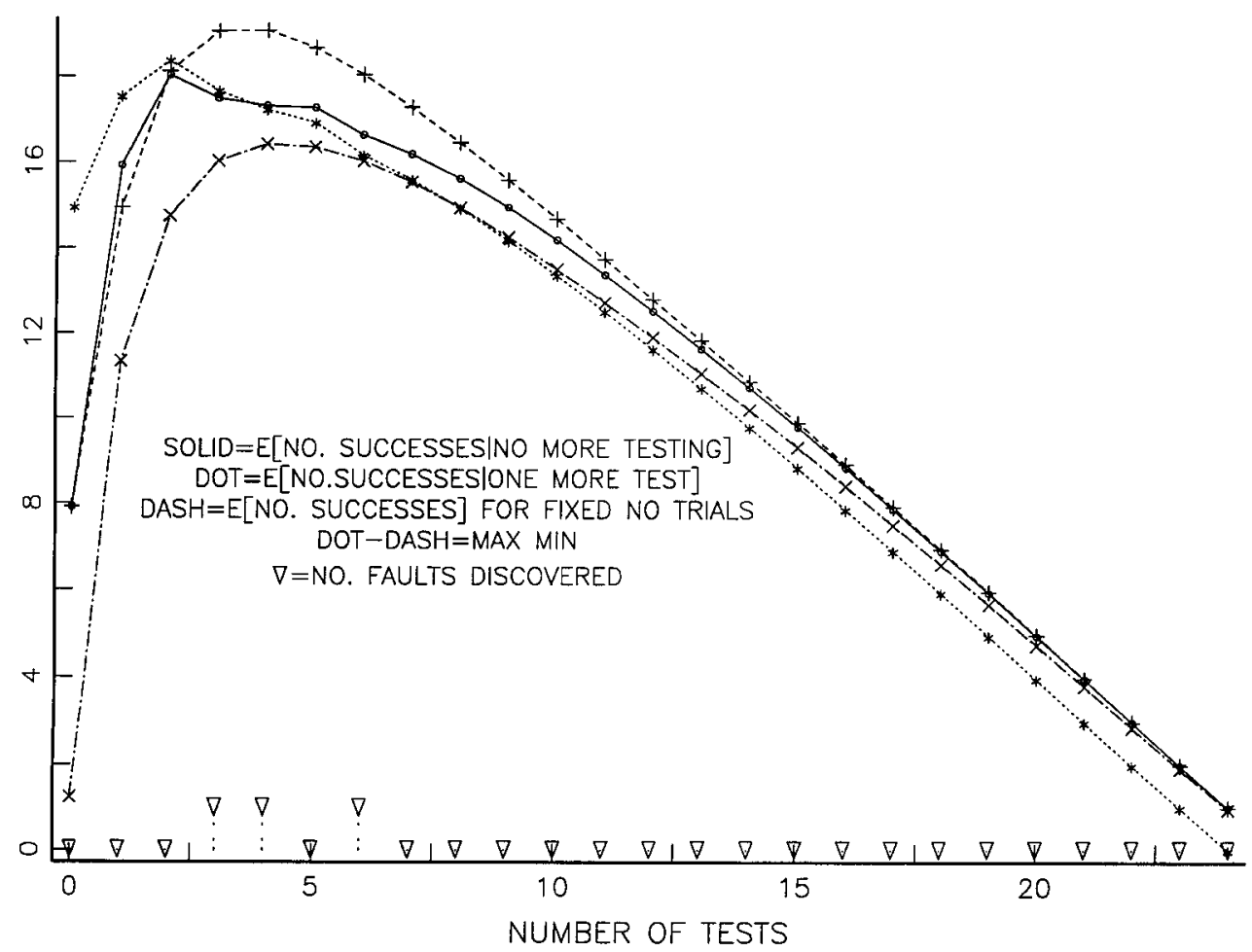

Figure 3. Number of faults $=3$, binomial discovery $p=.3$. Beta $A=1, B=1$ prior for $p$; faults prior Poisson 3.

number of faults is Poisson with mean 3 . The prior distribution for the discovery probability is uniform over $[0,1]$. The solid line displays the probability of all remaining missiles being successes as a function of the number of tests using the same fault discovery series and (3.19) using the posterior distribution of the discovery probability. The dotted line is the probability of all remaining missiles being successes as a function of the number of tests using the prior distribution of the discovery probability (fixed number of tests)

$$
P\left\{S_{t}=m-t\right\}=\int_{0}^{1} e^{-\lambda(1-p)^{t}\left[1-(1-p)^{m-t}\right]} f(p) d p .
$$

Consider the decision rule to test until the probability that all remaining missiles are successes is at least $\gamma$ for $\gamma=0.8$. For the uniform prior, the fixed number of tests calculation would test 6 . The Bayes calculation would test 12 . Both criteria recommend a larger number of tests than the expected number of successes criteria.

\section{DISCUSSION}

Our model directly addresses a real challenge faced by the testing community: to test efficiently with operational needs in mind. The present formulation is limited and simplified, but suggests the kinds of results to be expected, and that can be practically obtained. In 


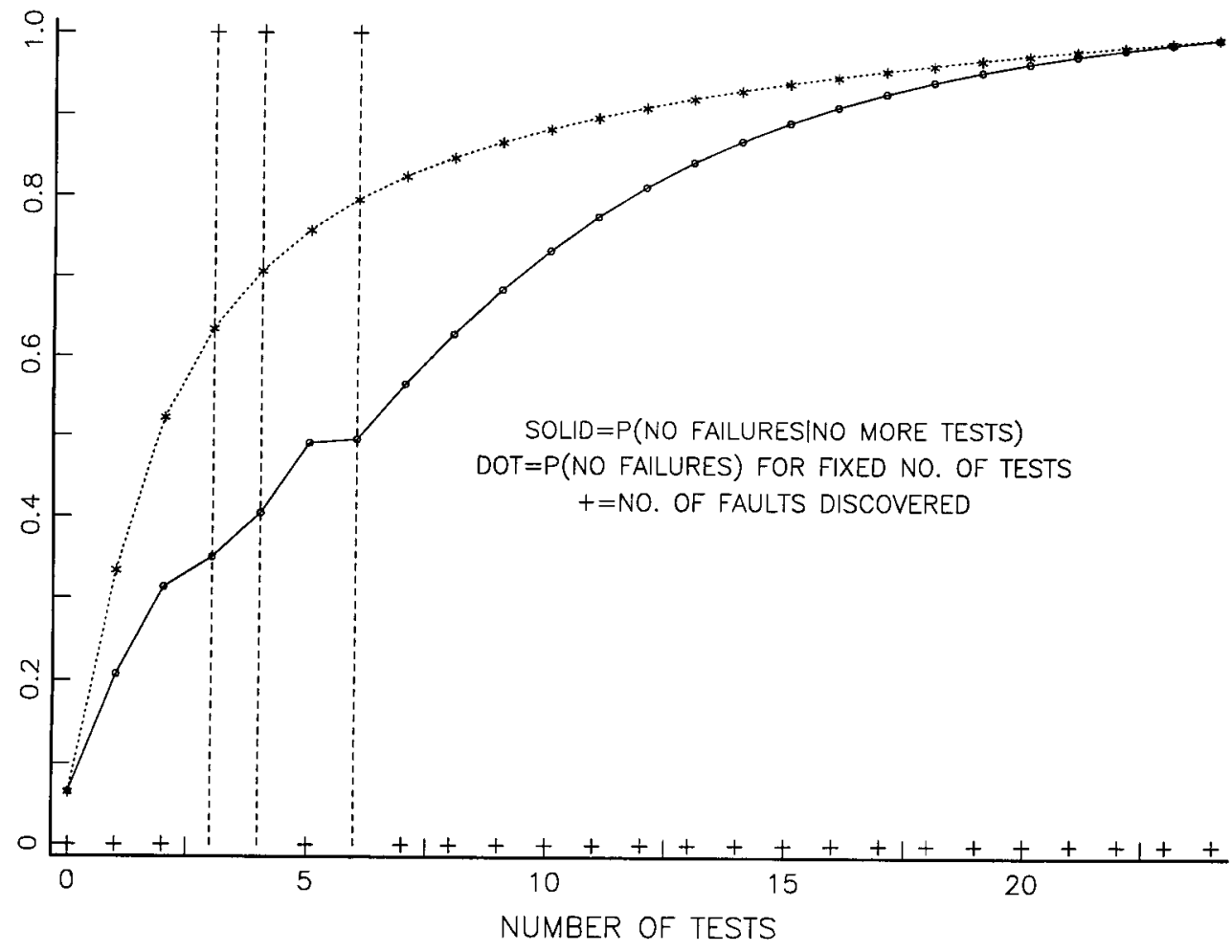

Figure 4. Number of faults $=3$, binomial discovery $p=.3$. Beta $A=1, B=1$ prior for $p$; faults prior Poisson 3.

particular the max-min approach (Section 2.4) provides a conservative assessment of a defensible conservative number of tests that one might consider making. This approach is quite robust to aspects of the model formulation (it actually accommodates different fault failure probabilities). The sequential myopic Bayes approach (Section 3) justifies adjustment of test effort to actual data obtained; it probably requires further detailed development before being practically applicable, but the needed modifications are understood, and are being made.

Implementation of the present approach requires a certain amount of computing, all within the range of desktop PCs or laptops. It is likely that user-friendly spreadsheet realizations of the current software can be developed.

\section{ACKNOWLEDGMENTS}

We are indebted to the Navy's COMOPTEVFOR, to DoD's DOT\&E, and to the Naval Postgraduate School for support and encouragement.

\section{REFERENCES}

[1] Ascher, H., and Feingold, H., Repairable Systems Reliability: Modeling, Inference, Misconceptions and Their Causes, Lecture Notes in Statistics, 7. Marcel Dekker, New York, 1984. 
[2] Balaban, H.S., "Reliability Growth Models," Journal of Environmental Sciences, 21, 11-18 (1978).

[3] Barlow, R.E., and Scheuer, E.M., "Reliability Growth During a Development Testing Program,'” Technometrics, 8, 53-60 (1966).

[4] Barr, D.R., “A Class of General Reliability Growth Models," Operations Research, 18, 5265 (1970).

[5] Bhattacharyya, G.K., Fries, A., and Johnson, R.A., ' $P$ Properties of Continuous Analog Estimators for a Discrete Reliability-Growth Model,' IEEE Transactions on Reliability, 38, 373-378 (1989).

[6] Calabria, R., Guida, M., and Pulcini, G., “'A Bayes Procedure for Estimation of Current System Reliability,', IEEE Transactions on Reliability, 41, 616-621 (1992).

[7] Chernoff, H., 'Sequential Models for Clinical Trials,' in Proceedings of the Fifth Berkeley Symposium on Mathematical Statistics and Probability, Vol. IV University of California Press, Berkeley, CA, 805-812 (1966).

[8] Chernoff, H., and Ray, S.N., “A Bayes Sequential Sampling Inspection Plan," Annals of Mathematical Statistics, 36, 1387-1407 (1965).

[9] Fries, A., "'Discrete Reliability-Growth Models Based on a Learning-Curve Property,' IEEE Transactions on Reliability, 42, 303-306 (1993).

[10] Gross, A.J., and Kamins, M., "Reliability Assessment in the Presence of Reliability Growth," in Annals of Assurance Sciences: 1968 Symposium on Reliability, 406-416 (1968).

[11] Hamming, R.W., Numerical Methods for Scientists and Engineers. McGraw-Hill, New York, (1973).

[12] IBM Corporation, A Graphical Statistical System (AGSS).

[13] Jayachadran, T., and Moore, L.R., "A Comparison of Reliability Growth Models," IEEE Transactions on Reliability, R-25, 49-51 (1976).

[14] Mazzuchi, T.A., and Soyer, R., "A Bayes Method for Assessing Product-Reliability During Development Testing,' IEEE Transactions on Reliability, 42, 503-510 (1993).

[15] Olsen, D.E., “'Estimating Reliability Growth,' IEEE Transactions on Reliability, R-26, 5053 (1977).

[16] Pollock, S.M., “A Bayesian Reliability Growth Model,, IEEE Transactions on Reliability, R17, 187-198 (1968).

[17] Woods, W.M., "The Effect of Discounting Failures and Weighting Data on the Accuracy of Some Reliability Growth Models,' in Proceedings of the Annual Reliability and Maintainability Symposium, Los Angeles, CA, Institute of Electrical and Electronics Engineers, Inc., 200-204, (1990).

[18] Yang, M.C., Wackerly, D.D., and Rosalsky, A., “Optimal Stopping Rules in Proofreading,' Journal of Applied Probability, 19, $723-729$ (1982).

Added in proof:

Huang, M-Y, Mcbeth, D., and Vardeman, S.B., "Development Test Programs for 1-shot systems: 2-State reliability and binary development-test results,' IEEE Transactions on Reliability, R-45, 379-385 (1996). 